\title{
Research on Data Acquisition Module Based on Rogowski Coil ECT
}

\author{
Yanhong Wang1, a, Wenxia Dü,b \\ Hebei Normal University, Shijiazhuang,China \\ Hebei Normal University, Shijiazhuang,China \\ wangjinhuisiling@163.com,dwx20040513@163.com
}

Keywords: Rogowski coil operational circuit integral link digital integrator

Abstract. Rogowski coil induction voltage is very small, and sometimes only a little or less. After the back of the circuit and other parts of the signal, the signal will almost decay to close to zero, and become very susceptible to external interference and distortion, which resulting in the back of the measurement circuit is difficult to measure the bus current value. So consider the integral link and Rogowski coil between the operational circuit to solve the above problems, while the integral part of the use of digital integrator.

The high-pressure sensing unit is located in the high-side side and is normally installed outdoors. The high-end sensing unit must be as simple as possible in order to meet its safety and reliability and ease of maintenance. The current microelectronics technology development, making it possible to design a technical solution to meet the demand.

\section{Operational circuit}

The general operational circuit is offset voltage, offset current, bias current, temperature drift and other issues. Through the accumulation of the back part of the accumulation of the entire measurement circuit will cause a great deviation. So we must use low-driftoperational circuit. Because the rogowski coil out of the signal is not DC component, just add a large impedance current limiting resistor as a protection circuit. This article uses LF365M as op amp device, its logic for the 2 feet and for the -IN, 3 feet for the + IN, 6 feet for the OUTPUT. The input resistance is high, which can be approved to reduce the amplifier circuit required for the sensor current. The output resistance Low, which can reduce the impact of load changes on the voltage gain. At the same time on the role of the current amplification, R3 from the current limiting effect. Circuit as shown below.

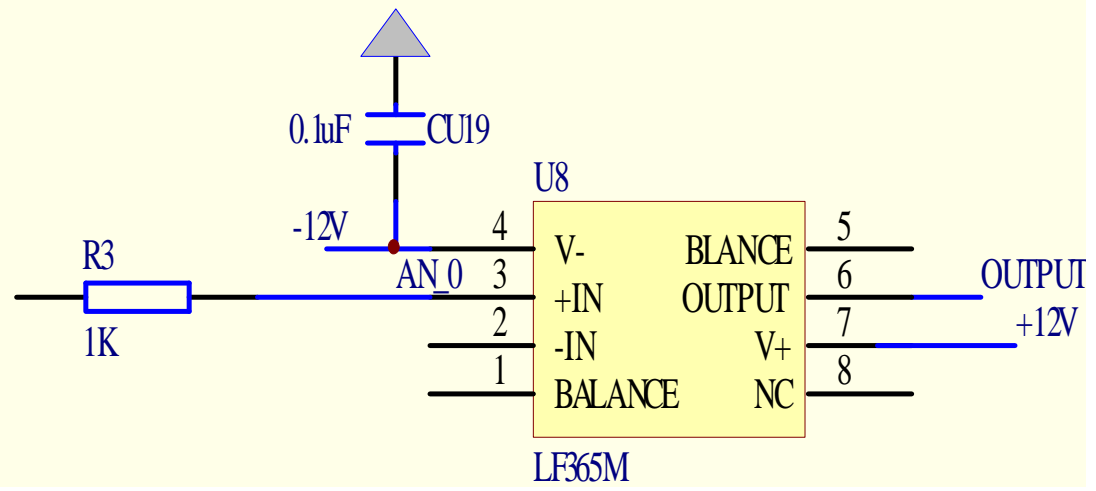

Fig. 1 operational circuit 


\section{Analog - to - digital conversion}

Using LF-398 sample to hold circuit, A / D converter using successive approximation 16-bit fast analog-to-digital converter AD7665, circuit design as shown in Figure 2:

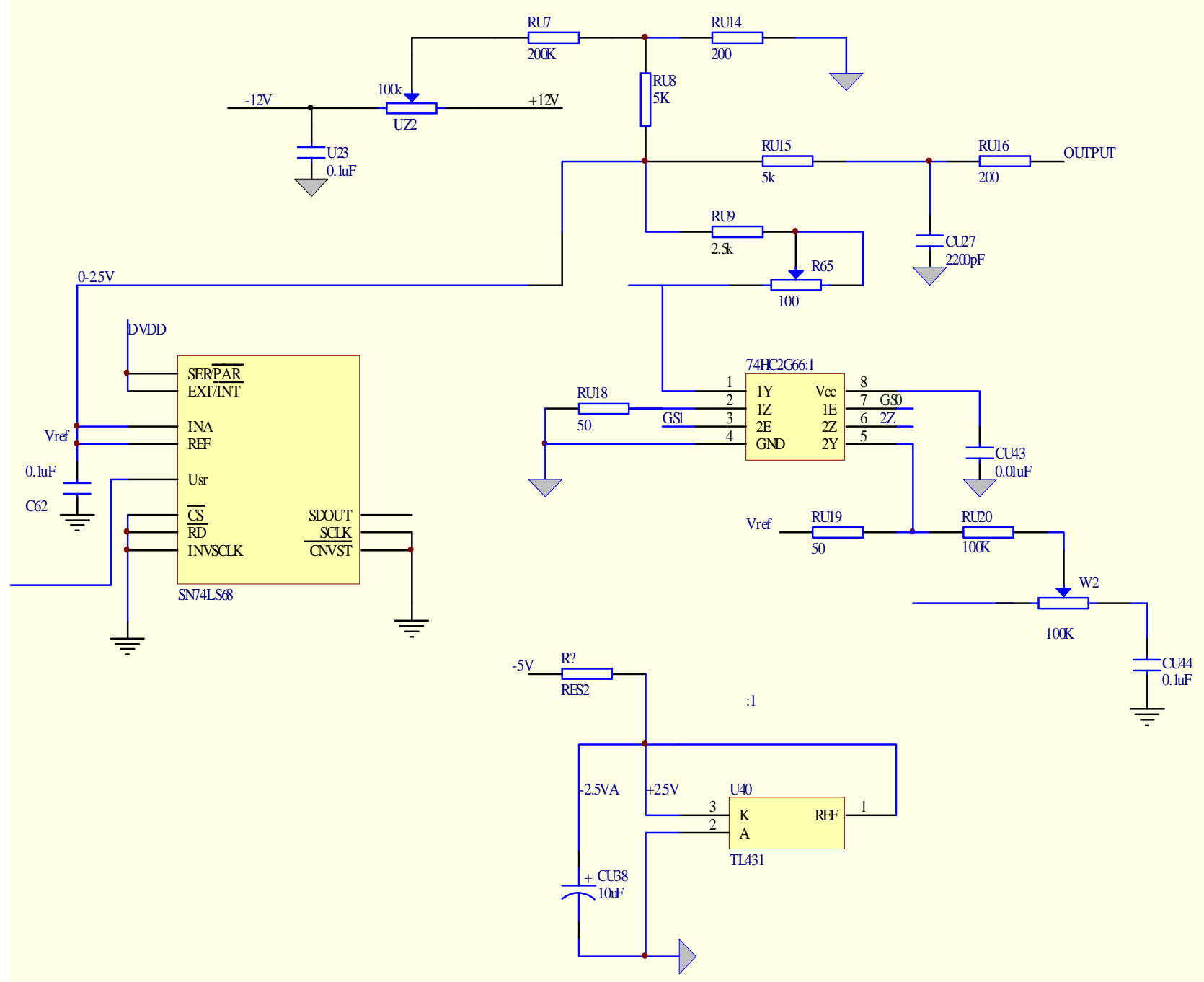

Fig. 2 Sampling and A / D conversion circuit

The AD7665 is a 16-bit fast analog-to-digital converter with successive conversion rates of 500KPS or 570KPS. The internal contains a high-speed 16-bit conversion circuit, an internal clock, suitable for different input range of the circuit, the output can be serial or parallel, serial interface 5V / $3 \mathrm{~V} .3$ terminal is the analog input; 8 terminal is the logic control input, when $=1$ for the sampling function, when $=0$ for the hold function; 5 terminal is the sample to keep the output;when $=1$ the output is serial, when $=0$ output In the parallel mode, and the text using the serial output mode; when= 1 the output data and SCLK to the input clock synchronization, for the open serial clock; and INVSCLK read the data and reduce the internal clock control signal; / D conversion input, the falling edge, the first trigger the internal sample and hold circuit to start A / D conversion; SDOUT for the serial data output, the first high to low output. The signal enters the resistor attenuation network through a single op amp and then performs A / D conversion. 


\section{Digital integrator}

With the emergence of high-speed digital signal processing technology, according to the sampling theorem, the low-frequency signal can be directly sampled, and then use the numerical method to restore the digital integration to be measured. This is the basis and basis for the digital integrator. When the sensor is used for measuring purposes. The measured frequency range is below kilohertz, and the digital integrator can be realized with a conventional high speed sampling system. Since the amount of voltage output from the sensor head is a differential form of the measured current in the low frequency range. The output of the sensor head can be directly integrated to obtain a signal proportional to the measured value.

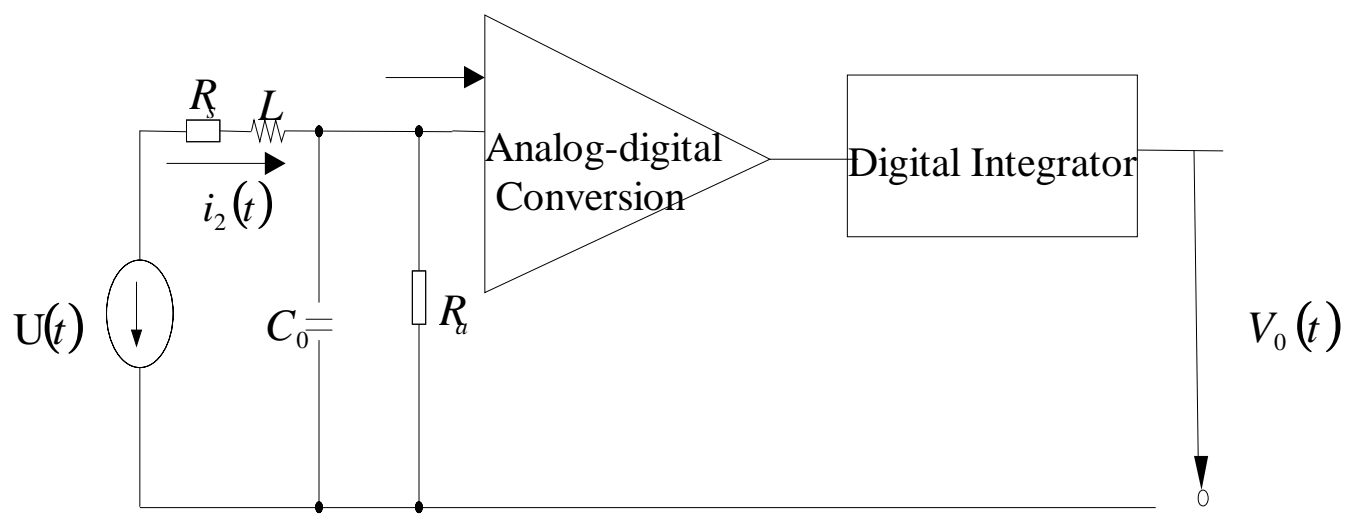

Fig.3 Schematic diagram of the digital integrator

The error of the Compound Multiplicative formula is the order, and then the composite trapezoidal Multiplicative formula is convergent. Since the measurement object of the power system is a sine wave and its harmonics, the integral interval is continuous and high order can be guided. So the use of digital integration is completely feasible.

At present, most of the use of analog-to-digital converters is used in the conversion The converter is to determine the range and the number of conversion bits (also reflects the conversion accuracy) on the basis of the input analog input directly into the corresponding digital An analog - to - digital converter. In order to ensure the conversion of the number of bits and conversion time under the premise of the direct converter in addition to quantitative error, the introduction of other sources of error, you can ensure that the signal is not distortion.

As the electronic sensor data acquisition requirements is a high-resolution, high sampling rate of the system, the general use of successive approximation. The basic components are: converters, high-speed voltage comparators and successive approximation logic registers. At present, the practical products are integrated into the above-mentioned parts of a single chip, and some even contain the reference voltage source, clock circuit, sample / hold, multi-channel analog switch and other auxiliary circuits to make it more independent, and function more complete.

Successive approximation completes a conversion with only one clock pulse (the number of bits for the converter). The conversion time is only related to the number of bits, regardless of the size of the input signal. The successive approximation of the sample conversion value is the instantaneous value. As long as the sampling rate satisfies the sampling theorem, the original signal can be completely reproduced by using the sampled value, and the spectral component is not distorted without considering the quantization error at the time of conversion.

The digital signal string obtained by the first integral re-conversion method is exactly the same as the original signal, except that a constant scale factor is added.

Successive approximation completes a conversion with only one clock pulse (the number of bits for the converter), the conversion time is only related to the number of bits, regardless of the size of the input signal. The successive approximation of the sample conversion value is the instantaneous value. 
As long as the sampling rate satisfies the sampling theorem, the original signal can be completely reproduced by using the sampled value, and the spectral component is not distorted without considering the quantization error at the time of conversion.

The digital signal string obtained by the first integral re-conversion method is exactly the same as the original signal, except that a constant scale factor is added.

\section{Conclusions}

This paper introduces the composition of each part of rogowski coil ECT, which focuses on the high side of the operational circuit, integral link, analog-to-digital link. It is found that the data acquisition module based on the rogowski coil ECT has been able to meet the needs of modern society.

\section{Acknowledgements}

This work was financially supported by the Hebei Science and Technology Self-prepare Project ( 15212115), and scientific research Project item of Hebei province education office under Grant (ZD2016053)

\section{References}

[1]P.N.Murgatroyd,D.N.Woodland,Geometrical properties of Rogowski sensors.IEE colloquium of low frequency power measurement and analysis. Savoy Place,2nd November 1994.

[2]Michie,W.C.,Cruden A.,Niewczas P,etc.Harmonic analysis of current waveforms using optical current sensor, Instrumentation and Measurement Technology Conference,2001.IMTC 2001.Proceedings of the 18th IEEE.Vol.3,21-23,May $2001: 1863-1865$.

[3] Yinxia Gao, Study on the Electronic Current Transducer, Yanshan University(2006)

[4]Adolescent $M$ etc.Optical current transducers for power Systems: A review〔J〕.IEEE Transept , 2004 , 19(4):1778-1788.

[5]M.Kanoi,G.Takahashi.Optic Voltage And Current Measuring System For Electric Power System.IEEE Trans.on Power Delivery,2006. 1(1):91-97 